Original Article

\title{
Application of Exploratory Factor Analysis (EFA) and Analytic Hierarchy Process (AHP) in Valuation of Urban Residential Land in Quoc Oai Town, Quoc Oai District, Hanoi City
}

\author{
Doan Quang Cuong, Tran Quoc Binh* \\ VNU University of Science, Vietnam National University, Hanoi, 334 Nguyen Trai, Hanoi, Vietnam
}

Received 29 November 2019

Revised 09 January 2020; Accepted 10 April 2020

\begin{abstract}
Land price is essential for land transactions, development of land policy,... Therefore, if the determination of land values is accurate and objective, it will greatly contributes to sustainable, economical and effective use of land resources. In this study, for identifying factors affecting land prices, we had interviewed 100 people, including land users, officials, real estate agents, land management experts. Using exploratory factor analysis, we identified five groups of 16 factors affecting land prices in Quoc Oai Town: location, policy, region, individuality, and environment, where location is the most influential factor group in land prices in Quoc Oai Town. Next, analytic hierarchy process is used to determine weight of each factor and calculate their influence index. The research results have proved the usefulness of combining EFA and AHP in identifying and quantifying factors influencing on land prices for land valuation.
\end{abstract}

Keywords: exploratory factor analysis, analytic hierarchy process, land price, Quoc Oai town.

\footnotetext{
* Corresponding author.

E-mail address: binh.geomatics@gmail.com

https://doi.org/10.25073/2588-1094/vnuees.4521
} 


\title{
Ứng dụng phương pháp phân tích nhân tố khám phá (EFA) và phân tích thứ bậc (AHP) trong định giá đất ở đô thị trên địa bàn thị trấn Quốc Oai, huyện Quốc Oai, thành phố Hà Nội
}

\author{
Đoàn Quang Cương, Trần Quốc Bình*
}

Truòng Đại học Khoa học Tụ nhiên, Đại học Quốc gia Hà Nội, 334 Nguyễn Trãi, Hà Nội, Việt Nam

Nhận ngày 29 tháng 11 năm 2019

Chỉnh sửa ngày 09 tháng 01 năm 2020; Chấp nhận đăng ngày 10 tháng 4 năm 2020

\begin{abstract}
Tóm tắt: Giá đất là cơ sở phục vụ cho các giao dịch về đất đai, xây dựng chính sách về đất đai,... Do vậy, nếu việc xác định giá trị đất đai được chính xác, khách quan thì sẽ góp phần quan trọng cho sử dụng đất đai bền vững, tiết kiệm và hiệu quả. Trong nghiên cứu này, để xác định các yếu tố ảnh hưởng đến giá đất, chúng tôi khảo sát 100 người dân (người dân sử dụng đất, cán bộ địa chính địa phường, nhân viên kinh doanh $\mathrm{BĐS}$, chuyên gia quản lý đất đai). Kết quả phân tích nhân tố khám phá cho thấy 5 nhóm gồm 16 yếu tố ảnh hưởng đến giá đất tại trị trấn Quốc Oai bao gồm vị trí, chính sách, khu vực, cá biệt, môi trường. Trong đó, vị trí là nhân tố có ảnh hưởng lớn nhất đến giá đất tại thị trấn Quốc Oai. Từ đó, trọng số của từng yếu tố được xác định bằng phương pháp phân tích đa chỉ tiêu (AHP) nhằm xây dựng chỉ số ảnh hưởng của các yếu tố đến giá đất. Kết quả nghiên cứu đã chứng minh tính hữu dụng của sự kêt hợp $\mathrm{EFA}$ và $\mathrm{AHP}$ trong nhận dạng và lượng hóa các yếu tố ảnh hưởng đến giá đất phục vụ công tác định giá đất.
\end{abstract}

Tù khoá: phân tích nhân tố khám phá, phân tích thứ bậc, giá đất, thị trấn Quốc Oai.

\section{Mở đầu}

Với tốc độ đô thị hóa nhanh như ở nước ta hiện nay, giá đất vùng ngoại ô các thành phố tăng nhanh chóng và là tâm điểm chú ý của các bên tham gia thị trường bất động sản. Chính vì vậy, xác định giá đất sát với giá trị thực sẽ giúp thị trường đất đai vận hành thông suốt. Để định giá đất chính xác cần xác định các yếu tố tác động đến giá đất như vị trí, khoảng cách đến các tiện ích công cộng, trung tâm hành chính, trường học,...

Thị trấn Quốc Oai là trung tâm huyện Quốc Oai, huyện ngoại thành nằm ở khu vực phía tây Thủ đô Hà Nội. Trong những năm gần đây, với

\footnotetext{
* Tác giả liên hệ.

Địa chỉ email: binh.geomatics@gmail.com

https://doi.org/10.25073/2588-1094/vnuees.4521
}

sự thay đổi mạnh mẽ về cơ sở hạ tầng và diện mạo đô thị, nông thôn, các giao dịch về bất động sản khu vực này ngày càng sôi động. Do đó, việc xác định các yếu tố hưởng đến giá đất là cơ sở để các nhà hoạch định chính sách có những giải pháp hiệu quả trong quy hoạch sử dụng đất và phát triển thị trường bất động sản.

Trên thế giới, việc xác định các yếu tố ảnh hưởng đến giá đất được thực hiện bằng nhiều phương thức khác nhau như phương pháp phân tích đa chỉ tiêu, mô hình hồi quy đa biến, mô hình hồi quy trọng số địa lý,... Bằng việc kết hợp trọng số và mức độ ảnh hưởng đến giá đất của các chỉ tiêu thành phần, bản đồ giá trị các nhóm yếu tố ảnh hưởng được xây dựng nhằm phục vụ định 
giá đất và cung cấp thông tin đất đai. Việc kết hợp phân tích hồi quy đa biến và phân tích địa thống kê đã được sử dụng nhằm xác định mức độ ảnh hưởng của các yếu tố đến giá đất trong nghiên cứu của Nzau [1]. Trong nghiên cứu của Demetris Demetrious [2], việc định giá đất được thực hiện bằng các kỹ thuật tiên tiến, bao gồm phân tích hồi quy bội (MRA) và phân tích trọng số địa lý bằng GIS để lựa chọn các nhân tố ảnh hưởng đến giá đất và xây dựng bản đồ giá đất. Với sự phát triển nhanh chóng của khoa học công nghệ, GIS còn có thể được kết hợp với trí tuệ nhân tạo nhằm nâng cao khả năng xử lý các vấn đề không gian [3]. Hiện nay, nhiều tác giả đã sử dụng tích hợp các mô hình thực nghiệm về giá đất chuẩn và GIS để đánh giá sự phát triển bền vững của đất ở đô thị. Ở nghiên cứu của Zhao Xu [4], các mô hình giá đất chuẩn được tích hợp với công nghệ GIS và trực quan hóa dữ liệu giá đất và mô phỏng không gian $3 \mathrm{D}$ cho các khu vực đất ở đô thị.

Tại Việt Nam, vấn đề giá đất được đề cập trong nhiều nghiên cứu. Việc xác định các mô hình, yếu tố ảnh hưởng đến giá đất trong nghiên cứu của Lê Khương Ninh [5], Nguyễn Quỳnh Hoa [6] được thực hiện dựa trên mô hình hồi quy tuyến tính. Tuy nhiên, việc xác định các yếu tố ảnh hưởng đến giá đất của các nghiên cứu này thường được đưa trực tiếp vào mô hình hồi quy và chưa có sự đánh giá sự phù hợp của các biến quan sát với đối tượng nghiên cứu. Với khả năng mô tả mối quan hệ giữa một tập hợp biến có thể quan sát được với các nhân tố không thể quan sát, EFA là phương pháp thích hợp để tìm ra các nhân tố thích hợp mới, chính xác và cập nhật [7]. Cùng với khả năng sắp xếp các chỉ tiêu theo mức độ quan trọng, từ đó tìm được quyết định cuối cùng hợp lý nhất, AHP là phương pháp thường được sử dụng để xác định mức độ ảnh hưởng của các yếu tố đến giá đất cho công tác định giá. Sự kết hợp GIS và phần mềm thống kê là công cụ mạnh trong thiết kế hệ thống định giá tự động bất động sản [3]. Do vậy, sự kết hợp của phương pháp phân tích nhân tố khám phá và phân tích thứ bậc được sử dụng cho nghiên cứu này.

\section{Các nhân tố ảnh hưởng đến giá đất}

Đất đai là yêu cầu cần thiết của tất cả các hoạt động kinh tế [8]. Về khía cạnh kinh tế, giá trị đất đai được hiểu là sự dự kiến về tính hữu dụng thông qua khả năng sử dụng nhằm đáp ứng các nhu cầu khác nhau của con người. Giá đất là một tham số để thể hiện giá trị của một đối tượng hoặc một tài sản. Giá thể hiện bằng tiền được chấp nhận chung để so sánh các giá trị trong thị trường [9]. Giá cả và giá trị đất đai được xác định không chỉ bởi vị trí của tài sản mà còn chịu tác động của nhiều yếu tố ngoại sinh và nội sinh trong thị trường [10]. Do đó, việc xác định giá đất cần dựa trên sự tác động tổ hợp của nhiều yếu tố đến giá đất. Trên thế giới, việc phân vùng giá đất phần lớn dựa trên mối quan hệ tương quan giữa giá đất và khoảng cách đến các khu trung tâm. Theo nghiên cứu của Liu Zheng, sự phát triển của giá đất được phân tích dựa trên khoảng cách tới các tiện ích công cộng, trường học. Nếu xét về góc độ các yếu tố tiếp cận, xác định giá đất còn được phát triển dựa trên khoảng cách giữa các thửa đất và các trung tâm thành phố, các trục giao thông chính, trung tâm văn hóa, y tế,... Ở tầm vĩ mô, giá đất chịu ảnh hưởng của các yếu tố kinh tế, nhân khẩu, môi trường trong đó các yếu tố có quan hệ chặt chẽ với giá trị đất đai bao gồm tỉ lệ nhập cư, thu nhập trung bình, lãi suất cho vay [11]. Một số yếu tố quan trọng nhất ảnh hưởng đến giá đất bao gồm tình trạng kinh tế, xã hội, môi trường, khoảng cách đến trung tâm hành chính, trung tâm thương mại, giao thông, các dịch vụ đô thị bao gồm công viên, sân thể thao, trung tâm y tế bệnh viện [12]. Dựa trên mô hình hồi quy trọng số địa lý, Nakamura chỉ ra chất lượng môi trường sống có tác động tích cực đến giá trị đất đai [13]. Tiếng ồn là một trong những nguồn gây khó chịu cho người dân, gây hại cho sức khỏe, giảm sự tập trung và hiệu quả công việc [14]. Do vậy, ảnh hưởng của tiếng ồn đến giá bất động sản được nghiên cứu ở nhiều nước phát triển. Becker (2003) đã phân tích sự ảnh hưởng của tiếng ồn ở những con đường lớn ở Israel, kết quả cho thấy giá trung bình của các bất động sản tăng $1,2 \%$ khi tiếng ồn giảm $1 \mathrm{~dB}$ 
[15]. Tương tự như vậy, dựa trên mô hình hedonic về giá đất, Kim và cộng sự đã chỉ ra rằng, tại khu vực đô thị Seoul, mức giá đất giảm $1,3 \%$ nếu tiếng ồn tăng lên $1 \%$ [16]. Tuy nhiên, tác động của tiếng ồn đến giá cả thường không ổn định và phụ thuộc vào đặc điểm của các khu vực khác nhau [17]. Bên cạnh yếu tố môi trường, giao thông vận tải có ý nghĩa quan trọng trong phát triển kinh tế, văn hóa, và có tác động không nhỏ đến giá đất. Sự hiện diện của hệ thống giao thông làm tăng giá trị đất đai do cải thiện khả năng tiếp cận. Mạng lưới giao thông tạo điều kiện cho hàng hóa lưu thông, giảm các chi phí vận tải, trong nghiên cứu của Demetrious mạng lưới giao thông (đường cao tốc, quốc lộ) có ảnh hưởng lớn đến giá trị đất đai [18]. Tại khu vực có đường quốc lộ, ga đường sắt đi qua, giá trị đất đai cao hơn từ $9,6 \%$ đến $10,1 \%$ so với các khu vực không có đường giao thông, đường sắt đi qua [19]. Ngoài các yếu tố mang tính chính sách, đặc điểm khu vực thì các yếu tố cá biệt như độ rộng mặt tiền, diện tích, hình dạng,... cũng có ảnh hưởng đáng kể đến giá đất [20]. Trong nghiên cứu của Shougeng $\mathrm{Hu}$, tác giả đánh giá mối quan hệ giữa giá đất và các yếu tố ảnh hưởng đến giá đất tại thành phố Vũ Hán, Trung Quốc, kết quả thể hiện mối quan hệ chặt chẽ giữa giá cả và diện tích đất [21]. Như vậy, có thể thấy rẳng, giá đất chịu ảnh hưởng của rất nhiều nhân tố khác nhau. Các nhân tố này có thể được chia dựa trên mối quan hệ của các yếu tố và phạm vi ảnh hưởng bao gồm: nhân tố vị trí, cá biệt, khu vực, chính sách,...

\section{Khu vực và phương pháp nghiên cứu}

\subsection{Khu vục nghiên cúu}

Thị trấn Quốc Oai nằm cách trung tâm TP.Hà Nội $20 \mathrm{~km}$ về phía tây. Với cơ sở hạ tầng giao thông được chú trọng đầu tư, những trục đường giao thông lớn được mở rộng và sự dịch chuyển của trụ sở các cơ quan hành chính. Theo quy hoạch đến năm 2030 định hướng 2050 khu vực phía tây của Hà Nội, trong đó có thị trấn Quốc Oai, sẽ trở thành khu vệ tinh mới của Hà Nội. Về hạ tầng giao thông, thị trấn Quốc Oai có nhiều tuyến đường huyết mạch đi qua, trong đó có đại lộ Thăng Long, quốc lộ 21A, tỉnh lộ 419 kết nối trung tâm thành phố với các dự án trọng điểm như khu công nghệ cao, khu đại học. Là thị trấn thuộc ngoại thành Hà Nội, tuy nhiên hiện nay tại thị trấn đã có hệ thống thu gom chất thải sinh hoạt, đảm bảo vệ sinh môi trường. Với vị trí địa lý nằm gần hai con sông là sông Tích và sông Đáy, nước ngầm khu vực thị trấn mạch nông khá dồi dào, trữ lượng lớn và $100 \%$ các hộ dân trong thị trấn được sử dụng nước sạch. Mặc dù có đường cao tốc lớn chạy qua nhưng tình trạng ô nhiễm không khí chỉ xảy ra cục bộ dọc trục Đại lộ Thăng Long, phía trong khu dân cư không khí trong lành. Ngoài ra, là trung tâm văn hóa - chính trị của cả huyện, thị trấn Quốc Oai là nơi tập trung các công trình kinh tế - xã hội. Trên địa bàn hiện nay có 4 trường học, nhà văn hóa quy mô $5000 \mathrm{~m}^{2}$, một bệnh viện đa khoa cấp III. Những điều kiện này góp thần thúc đẩy phát triển kinh tế - xã hội của thị trấn Quốc Oai nói riêng và huyện Quốc Oai nói chung.

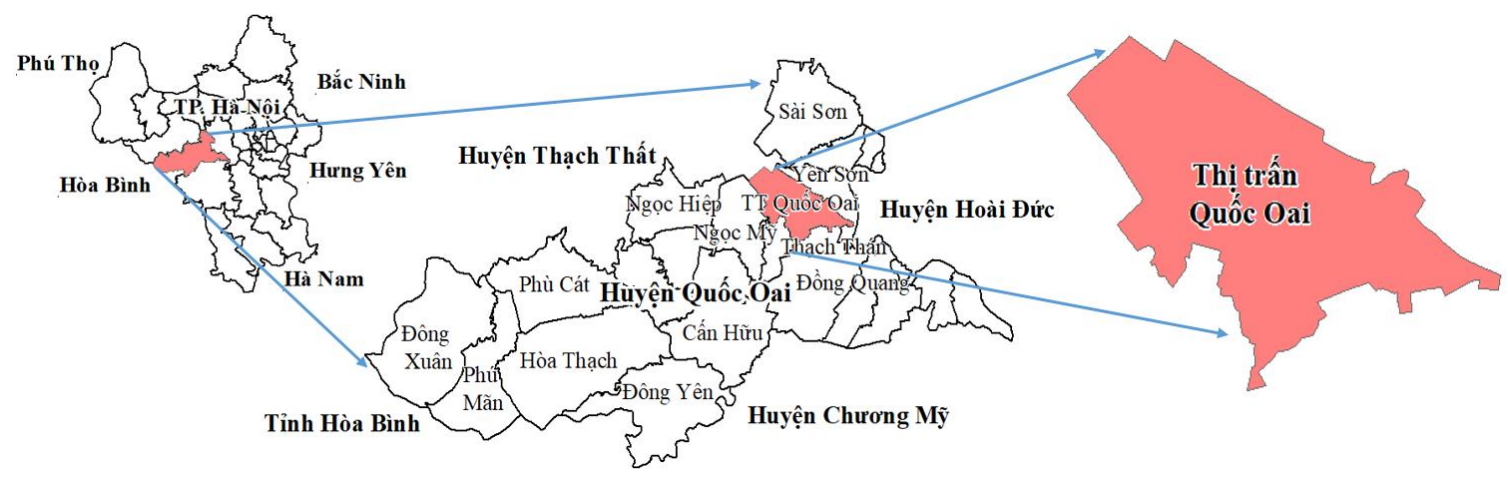

Hình 1. Sơ đồ vị trí của thị trấn Quốc Oai. 


\subsection{Phương pháp nghiên cưu}

Trước tiên, giả thuyết các nhân tố ảnh hưởng đến giá đất được nghiên cứu đặt ra dựa trên tài liệu khoa học. Sau đó, tiến hành lập bảng hỏi theo thang đo Likert và phỏng vấn người sử dụng đất, cán bộ quản lý đất đai,... Để đưa ra được các nhân tố ảnh hưởng đến giá đất, phương pháp phân tích nhân tố khám phá được sử dụng nhằm tìm ra các yếu tố có ảnh hưởng đến giá đất tại thị trấn Quốc Oai. Kết quả của phân tích nhân tố khám phá làm cơ sở cho việc phân tích đa chỉ tiêu từ đó tính toán chỉ số ảnh hưởng của các yếu tố ảnh hưởng, hỗ trợ công tác định giá đất. Nghiên cứu này đã sử dụng phần mềm ArcGIS để phân tích không gian và phần mềm SPSS để kiểm định thang đo và phân tích nhân tố khám phá.

\section{a. Phuơng pháp chọn mẫu nghiên cứu}

Để đảm bảo độ tin cậy cho phương pháp phân tích nhân tố khám phá, kích thước mẫu phải đảm bảo đủ lớn. Theo Hair, trong phân tích nhân tố khám phá, số lượng mẫu tối thiểu phải gấp 5 lần số biến quan sát [22]. Trong nghiên cứu này, giả thuyết đặt ra có 18 tham số cần ước lượng, do đó số lượng mẫu tối thiểu là $\geq 5 * 18=90$. Đế đảm bảo yêu cầu đặt ra, số lượng mẫu được lựa chọn trong nghiên cứu này là 100 mẫu quan sát. Đối với việc chọn mẫu, nghiên cứu tiến hành ngẫu nhiên bao gồm: người sử dụng đất, cán bộ quản lý đất đai, nhân viên kinh doanh bất động sản, chuyên gia trong lĩnh vực quản lý đất đai.

\section{b. Phưong pháp thu thập số liệu}

Để thu thập thông tin sơ cấp, nghiên cứu tiến hành thiết kế bảng hỏi. Nội dung bảng hỏi bao gồm các thông tin về người được hỏi và các nhân tố ảnh hưởng đến giá đất tại trị trấn Quốc Oai, huyện Quốc Oai, thành phố Hà Nội. Để khảo sát ý kiển về các yếu tố ảnh hưởng đến giá đất tại địa bàn nghiên cứu, chúng tôi sử dụng thang đo Likert với 5 mức độ: i) Ẩnh hưởng rất ít; ii) Ảnh hưởng ít; iii) Ảnh hưởng trung bình; iv) Ảnh hưởng nhiều; v) Ảnh hưởng rất nhiều. Ngoài các thông tin về đặc điểm thửa đất (diện tích, hình dạng, mặt tiền,...), giá giao dịch trong 2 năm gần đây, nội dung chính của phiếu hỏi là đánh giá về ảnh hưởng của 18 biến quan sát đến giá đất tại thị trấn Quốc Oai theo 4 nhóm thang đo tiềm năng: nhóm cá biệt, nhóm vị trí, nhóm khu vực, nhóm chính sách.

Nhóm cá biệt $(\mathrm{CB})$ gồm 5 biến quan sát: i) Diện tích của thửa đất (CB1), ii) Hình dạng của thửa đất (CB2), iii) Chiều dài mặt tiền (CB3), iv) Hướng của thửa đất (CB4), v) Loại đường tiếp giáp (CB5) (tham khảo theo Shougeng $\mathrm{Hu}$ [21]; Malaitham [19]; Demetrious [2]).

Nhóm vị trí (VT) gồm 6 biến quan sát: i) Khoảng cách đến ủy ban nhân dân (VT1), ii) Khoảng cách đến khu đô thị (VT2), iii) Khoảng cách đến chợ (VT3), iv) Khoảng cách đến bệnh viện (VT4), v) Khoảng cách đến trường học (VT5), vi) Khoảng cách đến khu công nghiệp (VT6) (tham khảo theo Bambang [23]; Phạm Sỹ Liêm [24]; Kheir, Nasr [11]; Demetrious [2]; Christopher R.Bollinger [25]; Bovkir [26]).

Nhóm khu vực (KV) gồm 4 biến quan sát: i) Môi trường nước (KV1), ii) Môi trường không khí (KV2), iii) Trình độ dân trí (KV3), iv) Tình trạng an ninh (KV4) (tham khảo theo Nakamura, Hiroki [13]; Nguyễn Quỳnh Hoa [6]).

Nhóm chính sách (CS) gồm 3 biến quan sát: i) Chính sách quy hoạch (CS1), ii) Chính sách kinh tế (CS2), iii) Chính sách thuế (CS3) (tham khảo theo Evren Ozus [27]; Kheir, Nasr [11]).

\section{c. Phương pháp xử lý, phân tích dũ liệu}

Dũ liệu sau khi được thu thập được đưa vào phần mềm SPSS để phân tích nhân tố khám phá. Quá trình xử lý, phân tích dữ bao gồm 2 bước chính.

Bước 1: Kiểm định thang đo bằng chỉ số Cronbach's Alpha.

Trong nghiên cứu, việc đo lường các nhân tố được thực hiện bằng nhiều câu hỏi quan sát. Do vậy, khi lập bảng hỏi, các biến quan sát là biến con của một nhân tố được tạo ra. Để đánh giá tính phù hợp của biến con với nhân tố mẹ, nghiên cứu tiến hành kiểm định thang đo bằng chỉ số Cronbach's Alpha. Chỉ số này sẽ giúp kiểm tra các biến quan sát của nhân tố mẹ có đáng tin cậy không, có tốt không, phép kiểm định này phản ánh mức độ tương quan chặt chẽ giữa các biến quan sát trong cùng một nhân tố. Chỉ số Cronbach's Alpha có giá trị biến thiên trong 
đoạn [0...1]. Theo lý thuyết, chỉ số này càng cao càng tốt, nhưng khi nó quá lớn $(>0,95)$ thì cho thấy có hiện tượng trùng lặp trong thang đo. Do đó, nếu chỉ số này lớn hơn 0,95 thì cần kiểm tra lại các biến trong thang đo. Theo Nguyễn Đình Thọ và Nguyễn Thị Mai Trang, các biến có hệ số tương quan biến tổng nhỏ hơn 0,3 sẽ bị loại $[28$, 29]. Nếu chỉ số Cronbach's Alpha $>0,6$ thì thang đo được cho là có độ tin cậy [30]. Như vậy, khi kiểm định thang đo, chỉ số Cronbach's Alpha nằm trong khoảng $[0,6-0,95]$ đảm bảo thang đo có độ tin cậy tốt.

Bước 2: Phân tích nhân tố khám phá EFA.

Mục tiêu chính của EFA nhằm mô tả mối quan hệ giữa một tập hợp các biến có thể quan sát được $\mathrm{k}$ (số lượng nhỏ hơn) với các biến không quan sát được [31]. Phân tích nhân tố hoạt động dựa trên nguyên tắc có thể đo lường được và giảm các biến có chung phương sai, không quan sát được [32]. Các tiêu chí trong phân tích nhân tố EFA: i) hệ số KMO (Kaiser- Meyer-Olkin) là chỉ số dùng để xem xét sự thích hợp của phân tích nhân tố. Trị số KMO phải đạt từ 0,5 trở lên $(0,5 \leq \mathrm{KMO} \leq 1)$. Nếu trị số này nhỏ hơn 0,5 thì phân tích nhân tố không phù hợp với tập dữ liệu nghiên cứu; ii) Kiểm định Bartlett (Bartlett's test of sphericity) dùng để xem xét các biến quan sát trong nhân tố có tương quan với nhau hay không. Kiểm định Bartlett có ý nghĩa thống kê nhỏ hơn 0,05 (sig Bartlett's Test $\leq 0,05$ ) chứng tỏ các biến quan sát có tương quan với nhau trong nhân tố; iii) Trị số Eigenvalue (trị riêng) là một tiêu chí sử dụng phổ biến để xác định số nhân tố trong phân tích EFA. Với tiêu chí này, chỉ những nhân tố có Eigenvalue $\geq 1$ mới được giữ lại trong mô hình phân tích; iv) Tổng phương sai trích (Total Variance Explained) $\geq 50 \%$ cho thấy các mô hình EFA là phù hợp. Trị số này thể hiện các nhân tố trích cô đọng được bao nhiêu \% và thất thoát bao nhiêu \% của các biến quan sát; v) Hệ số tải nhân tố cho biết mức độ đóng góp của biến quan sát với nhân tố. Theo Hair, nếu kích thước mẫu là 100 thì số hệ số tải nhân tố phải đảm bảo từ 0,55 trở lên.

\section{d. Phương pháp phân tích đa chỉ tiêu}

Trong đánh giá đa chỉ tiêu, vai trò của các nhân tố đối với giá đất là không giống nhau, do đó cần xác trọng số của từng nhân tố đối với giá đất. Trong bài báo này, việc xác định trọng số của các nhân tố được thực hiện bằng phương pháp phân tích đa chỉ tiêu, cụ thể là quá trình phân cấp thứ bậc AHP.

Phương pháp AHP được giới thiệu năm 1980 bởi Saaty [33]. AHP là kỹ thuật tạo quyết định, giúp sắp xếp các chỉ tiêu theo mức độ quan trọng, từ đó tìm được quyết định cuối cùng hợp lý nhất [33,34]. Cụ thể, quá trình phân tích phân cấp bao gồm 4 bước như sau: i) Xây dựng cấu trúc phân cấp, ii) Xây dựng ma trận so sánh của các chỉ tiêu, iii) So sánh cặp các chỉ tiêu theo mức độ quan trọng, iv) Tổng hợp và xác định trọng số cuối cùng. Về mặt bản chất, việc tính toán trọng số cho các chỉ tiêu khác nhau được thực hiện dựa trên so sánh cặp các yếu tố ảnh hưởng. Tầm quan trọng giữa hai tiêu chí được cho theo thang điểm từ 1 đển 9 để thể hiện sự quan trọng cao hơn (từ rất ít quan trọng đến quan trọng hơn rất nhiều) và các giá trị nghịch đảo từ $1 / 9$ đến 1 để thể hiện sự quan trọng thấp hơn. Trong quá trình phân tích AHP, quan hệ bắc cầu không được thiết lập khi so sánh cặp. Do đó, để kiểm tra mức độ nhất quán giữa các cặp so sánh, tỷ số nhất quán $(\mathrm{CR})$ được sử dụng: nếu $\mathrm{CR}<10 \%$ thì có thể chấp nhận được, ngược lại nếu $\mathrm{CR} \geq 10 \%$ thì cần kiểm tra lại các bước trước [33,34].

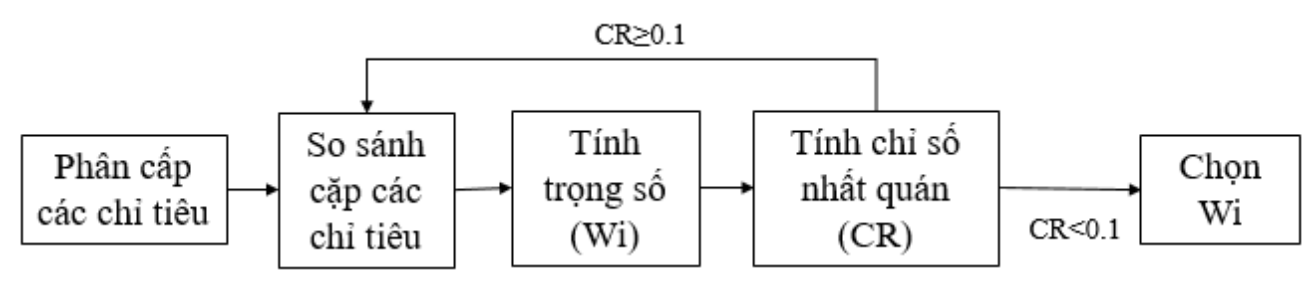

Hình 2. Quy trình xác định trọng số bằng phương pháp AHP. 


\section{Kết quả nghiên cứu và thảo luận}

\subsection{Xác định các yếu tố ảnh hương đến giá đất a. Kiểm định thang đo bằng Cronbach's Alpha}

Để đảm bảo các yếu tố ảnh hưởng đến giá đất tại thị trấn Quốc Oai có độ tin cậy, nghiên cứu đã tiển hành kiểm định thống kê qua chỉ số Cronbach Alpha. Qua kiểm định thang đo, kết quả cho thấy hệ số Cronbach Alpha của từng thang đo đều lớn hơn 0,6 . Trong đó, đặc điểm vị trí được đo lường bởi 5 biến quan sát và có giá trị Cronbach Alpha là cao nhất $(0,779)$ có nghĩa độ tin cậy của các biến quan sát trong nhóm này là cao nhất. Bên cạnh đó, hệ số tương quan biến tổng (Corrected item- total correlation) đều có giá trị $\geq 0,3$. Do đó không có biến quan sát nào bị loại. Kết quả này thể hiện, hệ thống thang đo đảm bảo chất lượng tốt với 18 biến quan sát.

Bảng 1. Tổng hợp kết quả phân tích chất lượng thang đo bằng hệ số Cronbach's Alpha

\begin{tabular}{|c|c|c|c|c|}
\hline STT & Thang đo & $\begin{array}{l}\text { Số } \\
\text { biến }\end{array}$ & $\begin{array}{l}\text { Biến } \\
\text { quan sát }\end{array}$ & $\begin{array}{l}\text { Cronbach } \\
\text { Alpha }\end{array}$ \\
\hline 1 & $\begin{array}{c}\text { Đặc } \\
\text { điểm } \\
\text { cá biệt }\end{array}$ & 5 & $\begin{array}{c}\text { CB1, CB2, } \\
\text { CB3, CB4, } \\
\text { CB5 }\end{array}$ & 0,667 \\
\hline 2 & $\begin{array}{l}\text { Đặc } \\
\text { điềm } \\
\text { vị trí }\end{array}$ & 6 & $\begin{array}{l}\text { VT1, VT2, } \\
\text { VT3, VT4, } \\
\text { VT5, VT6 }\end{array}$ & 0,779 \\
\hline 3 & $\begin{array}{c}\text { Đặc } \\
\text { điểm } \\
\text { khu vực }\end{array}$ & 4 & $\begin{array}{c}\text { KV1, } \\
\text { KV2, } \\
\text { KV3, KV4 }\end{array}$ & 0,664 \\
\hline 4 & $\begin{array}{l}\text { Chính } \\
\text { sách } \\
\text { nhà nước }\end{array}$ & 3 & $\begin{array}{l}\text { CS1, CS2 } \\
\text { CS3 }\end{array}$ & 0,727 \\
\hline
\end{tabular}

b. Phân tích nhân tố tố khám phá và lựa chọn yếu tố ảnh hưởng đến giá đất

Nhằm kiểm định các nhân tố trong thang đo được lựa chọn chính xác hay không, bằng phương pháp phương sai trích Principal component analysis, phương pháp xoay Varimax with Kaiser normalization, nghiên cứu tiến hành phân tích nhân tố khám phá, ở kết quả phân tích nhân tố lần 1 và lần 2, hai biến Hướng, $K C_{-} K C N$ có hệ số tải nhân tố < 0,55 vì vậy yếu tố này được loại ra khỏi mô hình. Sau khi chạy phân tích nhân tố lần thứ 3 , kết quả phân tích như sau:
Bảng 2. Bảng kiểm định KMO và Bartlett's Test

\begin{tabular}{|l|l|r|}
\hline \multicolumn{2}{|c|}{$\begin{array}{l}\text { Kaiser-Meyer-Olkin Measure of } \\
\text { Sampling Adequacy. }\end{array}$} & 0,632 \\
\hline $\begin{array}{l}\text { Bartlett's Test of } \\
\text { Sphericity }\end{array}$ & Approx. Chi-Square & 603,860 \\
\cline { 2 - 3 } & df & 120 \\
\cline { 2 - 3 } & Sig. & 0,000 \\
\hline
\end{tabular}

Kết quả kiếm định $\mathrm{KMO}=0,632 \geq 0,5$ cho thấy phân tích nhân tố khám phá phù hợp với tập dữ liệu nghiên cứu. Kiểm định tính tương quan giữa các biến quan sát cho thấy hệ số sig $=0,00$ (nhỏ hơn ngưỡng 0,05 như đã phân tích ở trên) cho thấy các biến quan sát có mối tương quan chặt chẽ trong mỗi nhân tố. Kết quả kiểm định phương sai trích: phương sai cộng dồn của các yếu tố là: $66,12 \%$. Như vậy, $66,12 \%$ thay đổi của các nhân tố được giải thích bởi các biến quan sát.

Kết quả phân nhóm các nhân tố cho thấy, hệ số tải nhân tố của các biến quan sát được giữ lại đều có giá trị lớn hơn 0,55 . Các giá trị này đảm bảo sự tương quan giữa biến quan sát và các nhân tố được tạo ra. Trong các biến quan sát được giữ lại ở nhóm vị trí, biến "khoảng cách đến bệnh viện" có hệ số tương quan là cao nhất. Như vậy, từ 4 nhóm nhân tố giả định ban đầu các biến quan sát đã được sắp xếp vào 5 nhóm (tương ứng với 5 cột): Nhóm vị trí (5 biến quan sát); nhóm chính sách (3 biến quan sát); nhóm đặc điểm cá biệt (4 biến quan sát); nhóm khu vực ( 2 biến quan sát); nhóm môi trường (2 biến quan sát).

Bảng 3. Ma trận xoay các nhân tố

\begin{tabular}{|c|c|c|c|c|c|}
\hline \multirow{2}{*}{} & \multicolumn{5}{|c|}{ Component } \\
\hline & 1 & 2 & 3 & 4 & 5 \\
\hline VT4 & 0,796 & & & & \\
\hline VT2 & 0,773 & & & & \\
\hline VT3 & 0,720 & & & & \\
\hline VT5 & 0,710 & & & & \\
\hline VT1 & 0,591 & & & & \\
\hline CS1 & & 0,873 & & & \\
\hline CS3 & & 0,843 & & & \\
\hline CS2 & & 0,643 & & & \\
\hline CB2 & & & 0,726 & & \\
\hline CB1 & & & 0,696 & & \\
\hline CB5 & & & 0,631 & & \\
\hline CB3 & & & 0,610 & & \\
\hline KV3 & & & & 0,887 & \\
\hline KV4 & & & & 0,867 & \\
\hline KV1 & & & & & 0,903 \\
\hline KV2 & & & & & 0,839 \\
\hline
\end{tabular}


4.2. Xác định trọng số, chỉ số các yếu tố ảnh hương phục vu định giá đất

Để xác định trọng số của từng nhóm nhân tố đến giá đất tại địa bàn, nghiên cứu tiến hành thiết lập ma trận so sánh mức độ quan trọng giữa các nhóm nhân tố. Từ kết quả đánh giá mức độ quan trọng của các nhân tố, tiến hành chuẩn hóa ma trận, tính toán giá trị $\lambda_{\max }$ và kiểm tra tỉ số nhất quán $\mathrm{CR}$. Trong trường hợp này, $\lambda_{\max }=5,16$, $\mathrm{CR}=0,04<0,1$. Do đó, sự nhất quán trong quá trình đánh giá được đảm bảo. Kết quả phân tích đa chỉ tiểu cho 5 nhóm nhân tố cho thấy, nhóm nhân tố vị trí và cá biệt ảnh hưởng mạnh nhất đến giá đất, với trọng số lần lượt là 0,41 và 0,27 .

Bảng 4. Trọng số các nhân tố ảnh hưởng đến giá đất

\begin{tabular}{|c|c|c|c|c|c|}
\hline & $\begin{array}{c}\text { Vị } \\
\text { trí }\end{array}$ & $\begin{array}{c}\text { Chính } \\
\text { sách }\end{array}$ & $\begin{array}{c}\text { Khu } \\
\text { vực }\end{array}$ & $\begin{array}{c}\text { Cá } \\
\text { biệt }\end{array}$ & $\begin{array}{c}\text { Môi } \\
\text { trường }\end{array}$ \\
\hline $\begin{array}{c}\text { Trọng } \\
\text { số }\end{array}$ & 0,41 & 0,15 & 0,09 & 0,27 & 0,08 \\
\hline
\end{tabular}

Đối với công tác định giá đất ở đơn vị cấp thị trấn, nhóm nhân tố chính sách mang tính vĩ mồ có thể được loại bỏ, bởi sự tác động của chúng trong cấp này là như nhau. Thị trấn Quốc Oai nằm ở khu vực ngoại thành thủ đô Hà Nội, phía đông giáp sông Đáy do đó nguồn nước dồi dào, $100 \%$ dân cư khu vực thị trấn được sử dụng nước sạch từ các nhà máy nước, môi trường nước đảm bảo. Ở phía Bắc thị trấn là khu công nghiệp Thạch Thất, loại hình khu công nghiệp này chủ yếu là lắp ráp linh kiện, ô nhiễm không khí chỉ mang tính chất cục bộ dọc đại lộ Thăng Long, khu vực dân cư không khí đảm bảo tốt. Do vậy, ảnh hưởng về môi trường khá hạn chế. Ngoài ra, thị trấn Quốc Oai là khu vực có trình độ dân trí cao nhất của huyện Quốc Oai, đây cũng là nơi có nhiều trụ sở an ninh. Vấn đề an ninh được đảm bảo tốt trong thị trấn. Do vậy, sự tác động của nhân tố môi trường, nhân tố khu vực đến giá đất tại thị trấn Quốc Oai là như nhau và có thể được đưa ra khỏi mô hình. Như vậy, để phục vụ công tác định giá đất, hai nhân tố vị trí (trọng số $60 \%$ ) và cá biệt (trọng số $40 \%$ ) với 9 yếu tố thành phần được đưa vào ma trận so sánh để xác định trọng số của từng yếu tố ảnh hưởng đến giá đất.

Kết quả phân tích phân cấp thể hiện rằng giá đất tại thị trấn Quốc Oai phần lớn chịu ảnh hưởng của các yếu tố loại đường tiếp giáp, khoảng cách đến các tiện ích như bệnh viện, trung tâm hành chính, chợ. Ngoài ra, trọng số của các yếu tố được sử dụng phục vụ định giá hàng loạt.

Bảng 5. Trọng số của các yếu tố trong nhóm vị trí được đánh giá bời 5 chuyên gia bằng $\mathrm{AHP}$

\begin{tabular}{|l|c|c|c|c|c|c|}
\hline & Phiếu 1 & Phiếu 2 & Phiếu 3 & Phiếu 4 & Phiếu 5 & Trọng số trung bình \\
\hline KC_TTHC (VT1) & 0,22 & 0,21 & 0,25 & 0,28 & 0,23 & 0,24 \\
\hline KC_KĐT (VT2) & 0,17 & 0,15 & 0,15 & 0,15 & 0,12 & 0,15 \\
\hline KC_Cho (VT3) & 0,26 & 0,30 & 0,25 & 0,25 & 0,33 & 0,27 \\
\hline KC_BV (VT4) & 0,17 & 0,18 & 0,18 & 0,20 & 0,17 & 0,18 \\
\hline KC_Truong (VT5) & 0,18 & 0,16 & 0,17 & 0,12 & 0,15 & 0,16 \\
\hline
\end{tabular}

Bảng 6. Trọng số của các yếu tố trong nhóm cá biệt được đánh giá bời 5 chuyên gia bằng $\mathrm{AHP}$

\begin{tabular}{|l|c|c|c|c|c|c|}
\hline & Phiếu 1 & Phiếu 2 & Phiếu 3 & Phiếu 4 & Phiếu 5 & Trọng số trung bình \\
\hline Diện tích $(\mathrm{CB} 1)$ & 0,12 & 0,10 & 0,12 & 0,11 & 0,09 & 0,11 \\
\hline Hình dạng (CB2) & 0,17 & 0,16 & 0,16 & 0,16 & 0,18 & 0,17 \\
\hline Mặt tiền $(\mathrm{CB} 3)$ & 0,26 & 0,28 & 0,24 & 0,23 & 0,27 & 0,25 \\
\hline Loại đường $(\mathrm{CB} 5)$ & 0,45 & 0,46 & 0,48 & 0,50 & 0,46 & 0,47 \\
\hline
\end{tabular}


Nghiên cứu tiến hành xây dựng chỉ số ảnh hưởng của các yếu tố đến giá đất tại thị trấn Quốc Oai thể hiện mức độ ảnh hưởng của từng yếu tố đến giá đất nói riêng và của từng nhóm nhân tố nói chung. Trong đó, chỉ số của từng yếu tố ảnh hưởng được xác định bằng tích giữa hệ số ảnh hưởng và trọng số ảnh hưởng đến giá đất, được xác định theo công thức sau: $I_{i j}=W_{i .}\left(K_{j} . W_{j}\right)$

Trong đó, $I_{i j}$ là chỉ số ảnh hưởng của yếu tố thứ $i$ ở phân cấp thứ $j$.

$W_{i}$ là trọng số ảnh hưởng của yếu tố $i$ được xác định theo ý kiến chuyên gia.

$K_{j}$ là hệ sổ ảnh hưởng của phân cấp thứ $j$ (được xác định bằng thương số giữa mức giá trung bình của từng khoảng chỉ tiêu với mức giá trung bình của khoảng chỉ tiêu có giá trị cao nhất).

$W_{j}$ là trọng số ảnh hưởng của phân cấp thứ $j$ được xác định theo ý kiến chuyên gia.

Cuối cùng, chỉ số ảnh hưởng của từng nhóm nhân tố được xác định bằng tổng chỉ số của các yếu tố thành phần trong nhóm nhân tố. Đây là chỉ số hỗ trợ định giá đất hàng loạt tại khu vực nghiên cứu.

$$
I_{T}=\sum_{k=1}^{n} I_{k}
$$

Trong đó: $I_{T}$ là chỉ số ảnh hưởng chung của các yếu tố thành phần, $I_{k}$ là chỉ số ảnh hưởng của yếu tố thứ $k, n$ là số yếu tố có ảnh hưởng đến giá đất.

Bảng 7. Chỉ số ảnh hưởng của các yếu tố trong nhân tố vị trí

\begin{tabular}{|c|c|c|c|c|c|c|c|c|c|}
\hline \multirow{3}{*}{$\begin{array}{l}\text { Yếu tố/ Trọng } \\
\text { số }\left(\mathrm{W}_{\mathrm{i}}\right)\end{array}$} & \multicolumn{9}{|c|}{ Phân cấp khoảng cách } \\
\hline & \multicolumn{3}{|c|}{$<500 \mathrm{~m}$} & \multicolumn{3}{|c|}{$500-1000 \mathrm{~m}$} & \multicolumn{3}{|c|}{$>1000 \mathrm{~m}$} \\
\hline & $\begin{array}{c}\text { Hệ số } \\
\left(\mathrm{K}_{\mathrm{j}}\right)\end{array}$ & $\begin{array}{c}\text { Troong số } \\
\left(\mathrm{W}_{\mathrm{j}}\right)\end{array}$ & $\begin{array}{c}\text { Chỉ số } \\
\left(\mathrm{I}_{\mathrm{ij}}\right)\end{array}$ & $\begin{array}{c}\text { Hệ số } \\
\left(\mathrm{K}_{\mathrm{j}}\right)\end{array}$ & $\begin{array}{c}\text { Trọng số } \\
\left(\mathrm{W}_{\mathrm{j}}\right)\end{array}$ & $\begin{array}{c}\text { Chỉ số } \\
\left(\mathrm{I}_{\mathrm{ij}}\right)\end{array}$ & $\begin{array}{c}\text { Hệ số } \\
\left(\mathrm{K}_{\mathrm{j}}\right)\end{array}$ & $\begin{array}{l}\text { Trọng số } \\
\left(\mathrm{W}_{\mathrm{j}}\right)\end{array}$ & $\begin{array}{c}\text { Chỉ số } \\
\left(\mathrm{I}_{\mathrm{ij}}\right)\end{array}$ \\
\hline $\begin{array}{l}\text { K/C đến bệnh } \\
\text { viện }(0,18)\end{array}$ & 1,0 & 0,58 & 0,103 & 0,82 & 0,29 & 0,042 & 0,74 & 0,13 & 0,018 \\
\hline $\begin{array}{c}\mathrm{K} / \mathrm{C} \text { đến trường } \\
\text { học }(0,16)\end{array}$ & 1,0 & 0,55 & 0,086 & 0,94 & 0,28 & 0,043 & 0,82 & 0,17 & 0,021 \\
\hline $\begin{array}{c}\text { K/C đến chợ } \\
(0,27)\end{array}$ & 1,0 & 0,56 & 0,152 & 0,84 & 0,30 & 0,067 & 0,74 & 0,14 & 0,028 \\
\hline $\begin{array}{c}\mathrm{K} / \mathrm{c} \text { dến trung } \\
\text { tâm hành chính } \\
(0,24)\end{array}$ & 1,0 & 0,54 & 0,129 & 0,93 & 0,31 & 0,068 & 0,75 & 0,15 & 0,027 \\
\hline $\begin{array}{l}\text { K/c đến khu đô } \\
\text { thị }(0,15)\end{array}$ & 1,0 & 0,54 & 0,079 & 0,83 & 0,29 & 0,036 & 0,78 & 0,17 & 0,020 \\
\hline
\end{tabular}

Bảng 8 . Chỉ số ảnh hưởng của các yếu tố trong nhân tố cá biệt

\begin{tabular}{|c|c|c|c|c|c|}
\hline \multicolumn{2}{|c|}{ Yếu tố / Trọng số $\left(\mathrm{W}_{\mathrm{i}}\right)$} & Phân cấp yếu tố & Trọng số $\left(\mathrm{W}_{\mathrm{j}}\right)$ & Hệ số $\left(K_{\mathrm{j}}\right)$ & Chỉ số $\left(\mathrm{I}_{\mathrm{ij}}\right)$ \\
\hline \multirow{3}{*}{\multicolumn{2}{|c|}{$\begin{array}{c}\text { Diện tích } \\
(0,11)\end{array}$}} & $<50 \mathrm{~m}^{2}$ & 0,18 & 0,74 & 0,014 \\
\hline & & $50-100 \mathrm{~m}^{2}$ & 0,56 & 1,0 & 0,062 \\
\hline & & $>100 \mathrm{~m}^{2}$ & 0,26 & 0,9 & 0,026 \\
\hline \multirow{6}{*}{$\begin{array}{c}\text { Hình dạng } \\
(0,17)\end{array}$} & \multirow{3}{*}{$\begin{array}{l}\text { Sổ đỉnh } \\
(0,67)\end{array}$} & 4-6 đỉnh & 0,54 & 1,0 & 0,060 \\
\hline & & 7-9 đỉnh & 0,32 & 0,95 & 0,033 \\
\hline & & $>9$ dỉnh & 0,14 & 0,92 & 0,015 \\
\hline & \multirow{3}{*}{$\begin{array}{c}\text { Độ rộng góc } \\
\quad(0,33)\end{array}$} & $<20^{\circ}$ & 0,54 & 1,0 & 0,030 \\
\hline & & $20-40^{\circ}$ & 0,31 & 0,87 & 0,015 \\
\hline & & $>40^{\circ}$ & 0,15 & 0,80 & 0,006 \\
\hline \multirow{3}{*}{\multicolumn{2}{|c|}{$\begin{array}{l}\text { Loại đường } \\
\quad(0,47)\end{array}$}} & Loại 2 & 0,73 & 1,0 & 0,345 \\
\hline & & Loại 5 & 0,17 & 0,66 & 0,052 \\
\hline & & Loại 6 & 0,10 & 0,57 & 0,026 \\
\hline \multirow{3}{*}{\multicolumn{2}{|c|}{$\begin{array}{c}\text { Mặt tiền } \\
(0,25)\end{array}$}} & $<5 \mathrm{~m}$ & 0,15 & 0,81 & 0,029 \\
\hline & & $5-10 m$ & 0,30 & 0,90 & 0,070 \\
\hline & & $>10 \mathrm{~m}$ & 0,55 & 1,0 & 0,141 \\
\hline
\end{tabular}


Với mục tiêu xây dựng bản đồ giá đất, nghiên cứu tiến hành thu thập dữ liệu về giá thị trường của các thửa đất nằm trong khu vực. Trong nghiên cứu này, giá đất tại thị trấn Quốc Oai chịu ảnh hưởng từ 9 yếu tố thành phần và được thể hiện thông qua chỉ số nhân tố chung (là giá trị trung bình trọng số của chỉ số nhân tố vị trí và nhân tố cá biệt). Như vậy, giá đất thị trường tại thị trấn Quốc Oai được xác định dưới dạng sau:

$$
G=I_{T}^{*} G_{Q C}
$$

Trong đó: $G$ là giá đất của thửa đất cần xác định, $I_{T}$ là chỉ số ảnh hưởng chung, $G_{Q C}$ là giá quy chuẩn (đã được tách các yếu tổ ảnh hưởng khỏi giá đất). Giá quy chuẩn $G_{Q C}$ được xác định là thương số giữa giá đất thu thập được và chỉ số ảnh hưởng chung $\left(I_{T}\right)$.

Giá trị $G_{Q C}$ của toàn bộ khu vực được xác định bằng phương pháp nội suy giá của 126 điểm giá thu thập trên thị trường. Trong nghiên cứu này, phương pháp nội suy trị trung bình trọng số (IDW) được sử dụng để nội suy giá đất, vì trong ba phương pháp nội suy IDW, Kriging, Spline thì phương pháp IDW thường cho kết quả tốt hơn [35]. Để tính giá đất quy chuẩn trung bình cho các thửa đất, công cụ Zonal Statistic as Table được sử dụng, giá đất quy chuẩn trung bình của các thửa đất thu được ở trường MEAN. Mỗi thửa đất riêng biệt có chỉ số ảnh hưởng của các yếu tố là khác nhau, do đó để tính giá đất cho các thửa đất, nghiên cứu tính giá đất cho từng thửa đất. Giá đất này được xác định bằng tích giữa giá quy chuẩn trung bình và chỉ số ảnh hưởng chung của thửa đất đó. Kết quả thu được bản đồ giá đất tại thị trấn Quốc Oai, với giá đất trung bình là 13,54 triệu đồng $/ \mathrm{m}^{2}$, giá cao nhất dao động từ $27-28$ triệu đồng $/ \mathrm{m}^{2}$ thuộc khu vực trung tâm thị trấn. Các thửa đất nằm trong các làng mức giá chỉ từ 7 triệu đồng $/ \mathrm{m}^{2}$.

\section{3. Đánh giá độ tin cậy của kết quả}

Để so sánh mức độ phù hợp của chỉ số ảnh hưởng của các yếu tố đến giá đất của khu vực thị trấn Quốc Oai. Tác giả lựa chọn phương pháp hồi quy trọng số địa lý (Geographically Weighted Regression) để đối sánh với kết quả giá đất xây dựng từ bộ chỉ số ảnh hưởng. Hồi quy theo trọng số địa lý (GWR) là kỹ thuật hồi quy cục bộ được sử dụng để ước tính mối quan hệ giữa biến độc lập và biến phụ thuộc theo không gian [36]. Trong lĩnh vực định giá đất, đây là phương pháp được sử dụng phổ biến nhằm xác định mức độ đóng góp của biến độc lập đối với biến phụ thuộc. Ngoài việc thể hiện mối quan hệ giữa các biến, mô hình GWR còn cho phép giải thích mối quan hệ giữa biến phụ thuộc và các biến độc lập thay đổi theo không gian. Kết quả mô hình hồi quy trọng số địa lý của 126 giá thu thập, với biến phụ thuộc là giá đất, biến độc lập là chỉ số vị trí (I_vitri), chỉ số cá biệt (I_cabiet) như sau:

Bảng 9. Kết quả hồi quy GWR

\begin{tabular}{|c|c|c|c|}
\hline Biến & $\begin{array}{c}\text { Hệ số } \\
\text { chặn }\end{array}$ & I_vitri & I_cabiet \\
\hline Hệ số hồi quy & 6,06 & 20,74 & 14,08 \\
\hline Giá trị phần dư & \multicolumn{3}{|c|}{0,01} \\
\hline $\mathrm{R}^{2}$ hiệu chỉnh & \multicolumn{3}{|c|}{0,8115} \\
\hline
\end{tabular}

Để đánh giá hiệu suất của mô hình GWR, chỉ số $\mathrm{R}^{2}$ hiệu chỉnh được sử dụng. $\mathrm{R}^{2}$ đại diện cho hệ số xác định và đo lường mức độ phù hợp của dữ liệu, giá trị $\mathrm{R}^{2}$ có giá trị nằm trong khoảng [0;1] [37]. Giá trị $\mathrm{R}^{2}$ hiệu chỉnh là 0,8115 cho thấy $81,15 \%$, giá đất được tại thị trấn Quốc Oai được giải thích bởi các biến trong mô hình. Hệ số phần dư là 0,01 gần với giá trị 0 , có nghĩa sự khác biệt giữa giá trị quan sát và giá ước tính là rất ít. Kết quả thể hiện mô hình phù hợp với tập dữ liệu nghiên cứu. Như vậy, mô hình giá đất ở đô thị tại thị trấn Quốc Oai được xác định bởi công thức:

$$
P=6,06+20,74 . \text { Vitri }+14,08 \text {. Cabiet }+0,01
$$

Kết quả hồi quy $\beta$ cho thấy, khi giá trị chỉ số vị trí tăng lên 0,1 đơn vị giá đất ở đô thị tại thị trấn Quốc Oai tăng 2,074 triệu đồng $/ \mathrm{m}^{2}$ và ngược lại. Khi giá trị chỉ số cá biệt tăng 0,1 đơn vị, giá đất ở đô thị tại thị trấn Quốc Oai tăng 1,408 triệu đồng $/ \mathrm{m}^{2}$. Theo kết quả hệ số hồi quy, nhân tố vị trí $(\beta=20,74)$ là nhân tố có mức độ ảnh hưởng rất cao đến giá đất ở đô thị ở thị trấn Quốc Oai, nhân tố cá biệt có hệ số $\beta=14,08$. So sánh tỉ lệ ảnh hưởng của hai nhân tố này đến giá đất với trọng số xác định bằng phương pháp phân tích 
thứ bậc (AHP), kết quả cho thấy sự chênh lệch không đáng kể khi xác định bởi hai phương pháp.

Sử dụng kết quả mô hình hồi quy trọng số địa lý để định giá đất cho khu vực thị trấn Quốc Oai, kết quả thu được giá đất thị trường của từng thửa đất. So sánh mức độ chênh lệnh về giá đất thực hiện bằng phương pháp sử dụng chỉ số ảnh hưởng của yếu tố và phương pháp GWR, có thể thấy rằng, kết quả của hai phương pháp khá tốt, mức độ chênh lệch không nhiều. Giá trị độ lệch trung bình là 0,004 triệu đồng $/ \mathrm{m}^{2}$, độ lệch chuẩn là 1,64 triệu đồng $/ \mathrm{m}^{2}$. Trong đó số thửa có mức chênh lệch nhỏ hơn 1,64 triệu đồng $/ \mathrm{m}^{2}$ chiếm $72,5 \%$, số thửa có mức chênh lệch nhỏ hơn 2,5 triệu đồng $/ \mathrm{m}^{2}$ chiếm tỉ lệ $89,01 \%$ tổng số thửa được định giá.

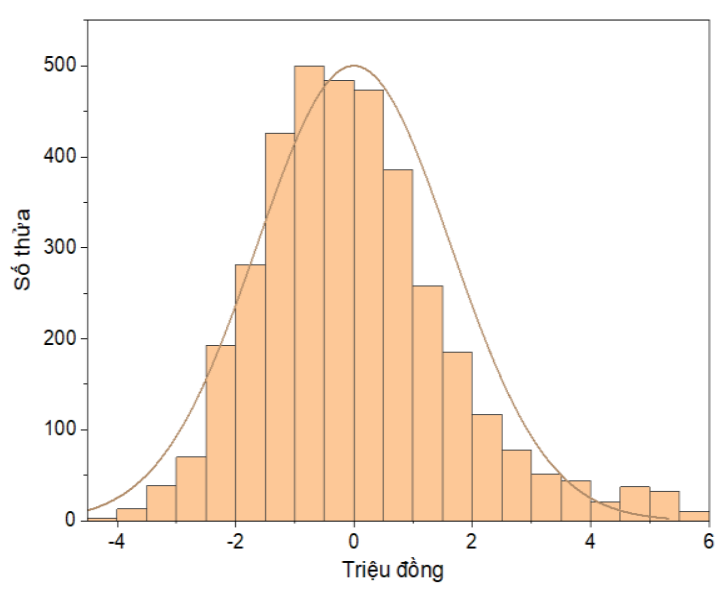

Hình 3. Biểu đồ phân bố chênh lệch giá đất của hai phương pháp.

\section{Kết luận}

Từ dữ liệu điều tra khảo sát thực địa về các yếu tố ảnh hưởng đến giá đất tại khu vực nghiên cứu, phương pháp phân tích nhân tố khám phá (EFA) và hệ thống thông tin địa lý (GIS) có khả năng đánh giá thang đo và phân nhóm các nhân tố ảnh hưởng đến giá đất. Qua kết quả phân tích nhân tố khám phá về các yếu tố ảnh hưởng đến giá đất tại thị trấn Quốc Oai, 5 nhóm nhân tố có ảnh hưởng đến giá đất bao gồm: vị trí, chính sách, cá biệt, khu vực, môi trường. Trong đó, 2 nhân tố được đánh giá quan trọng nhất đối với giá đất ở đô thị là nhân tố vị trí $(0,41)$ và nhân tố cá biệt $(0,27)$. Ngoài ra, phương pháp phân tích đa chỉ tiêu AHP được sử dụng nhằm xác định trọng số của các chỉ tiêu thành phần trong các nhóm nhân tố. Trọng số của các yếu tố này được đưa vào quá trình xây dựng chỉ số ảnh hưởng của các yếu tố đến giá đất, phục vụ công tác định giá đất hàng loạt. Kết quả kiểm định thể hiện giá đất xác định bằng chỉ số ảnh hưởng có sự chênh lệch ít so với phương pháp GWR, với $89,01 \%$ chênh lệch dưới 2,5 triệu đồng $/ \mathrm{m}^{2}$.

\section{Tài liệu tham khảo}

[1] Nzau, Modelling of influecen of urban sub-centres on spatial and temporal urban land value pattern, Case study of Nairobi, Kenya, Enschede, The Netherlands: International Institute for Aerial Survey and Earth Sciences (ITC), 2003.

[2] D.Demetris, The assessment of land valuation in land consolidation schemes: The need for a new land valuation framework, Land Use Policy 54 (2016) 487-498. https://doi.org/10.1016/ j.landusepol. 2016.03.008.

[3] G.Noelia, M.Gámez, E.Alfaro, ANN+ GIS: An automated system for property valuation, Neurocomputing 71 (2008) 733-742. https:// doi.org/10.1016/j.neucom.2007.07.031.

[4] X.Zhao, Q.Li, Integrating the empirical models of benchmark land price and GIS technology for sustainability analysis of urban residential development, Habitat International 44 (2014) 79-92. https://doi.org/10.1016/j.habitatint.2014. 04.012.

[5] L.K. Ninh, Factors affecting the price of periurban land in the Mekong Delta, Journal of Asian Business and Economic Studies 254 (2011) 11-17 (in Vietnamese).

[6] N.Q.Hoa, N.H. Duong, Building a model of mass land valuation for Go Vap district, Ho Chi Minh City, Journal of Asian Business and Economic Studies 26(3) (2016) 82-103 (in Vietnamese).

[7] F.L. Paulo, P. Belfiore, Principal Component Factor Analysis, Multivariate Exploratory Data Analysis, Elsevier Inc., 2019, pp.383-438.

[8] J.B. Nichols., S.D. Oliner, M.R. Mulhall, Swings in commercial and residential land prices in the United States, Journal of Urban Economics 73(1) (2013) 57-76, https://doi.org/10.1016/j.jue. 2012. 06.004 . 
[9] V.Willy, Land value and price of land, Land use, land cover and soil sciences 3, UNESCO-EOLSS, 2005.

[10] G. George, P. Tatian, K. Pettit, Supportive Housing and Neighborhood Property Value Externalities, Land Economics 80 (2004) 33-54. https://doi.org/10.2307/3147143.

[11] K. Nasr, B.A. Portnov, Economic, demographic and environmental factors affecting urban land prices in the Arab sector in Israel, Land Use Policy 50(2016)518-527.https://doi.org/ 10.1016/j.landusepol. 2015. 08.031.

[12] M. Jafar, R. Samadi, A. Hosseini, Evaluating and analysis of socio-economic variables on land and housing prices in Mashhad, Iran, Sustainable Cities and Society 41 (2018) 695-705. https:// doi.org/10.1016/j.scs.2018.06. 022.

[13] N. Hiroki, Relationship among land price, entrepreneurship, the environment, economics, and social factors in the value assessment of Japanese cities, Journal of Cleaner Production 217 (2019) 144-152. https://doi.org/10.1016/j.jclepro. 2019.01.201.

[14] WHO, Burden of Disease From Environmental Noise The World Health Organization, 2011.

[15] B. Nir, D. Lavee, The Benefits and Costs of Noise Reduction, Journal of Environmental Planning and Management 46 (2003) 97-111. https://doi. org/10.1080/713676703.

[16] K.K. Sik, S.J. Park, Y.J. Kweon, Highway traffic noise effects on land price in an urban area, Transportation Research Part D: Transport and Environment 12 (4) (2007) 275-280. https://doi. org/10.1016/j.trd.2007.03.002.

[17] M.D. Carlos, Does noise have a stationary impact on residential values?, Journal of European Real Estate Research 2(3) (2009) 259-279. https://doi. org/10.1108/1753926091099 9992.

[18] D.M. Faris, Estimating land value uplift around light rail transit stations in Greater Kuala Lumpur: An empirical study based on geographically weighted regression (GWR), Research in Transportation Economics 74 (2019) 10-20. https:// doi.org/10.1016/j.retrec. 2019.01. 003.

[19] M. Sathita, A. Fukuda, V. ichiensan, V. Wasuntarasook (2018), Hedonic pricing model of assessed and market land values: A case study in Bangkok metropolitan area, Thailand, Case Studies on Transport Policy, Available online 21
September 2018. https://doi.org/10.1016/j.cstp. 2018.09.008

[20] H.V. Dung, Identify factors that affect real estate prices, Journal of Finance 11 (2016) 54-57 (in Vietnamese).

[21] H. Shougeng, S. Yang, W. Li, C. Zhang, F. Xu, Spatially non-stationary relationships between urban residential land price and impact factors in Wuhan city, China, Applied Geography 68 (2016) 48-56. https://goi.org/10.1016/j.apgeog. 2016.01. 006.

[22] J. Hair, W.C. Black, B.J. Babin, R.E. Anderson, Multivariate Data Analysis, Pearson, 2009.

[23] B. Shenavr, S.M. Hosseini, Comparison of multicriteria evaluation (AHP and WLC approaches) for Land capability assesment of Urban development in GIS, International Journal of geomatics and geosciences 4 (2014) 435-446.

[24] P.S. Liem, T.V. Tuan, Building Land Price Zone in Rural Areas: A Case Study of Duong Quang Commune, Gia Lam District, Hanoi City, Vietnam Journal Agriculture Science 16 (2018) 601-612 (in Vietnamese).

[25] C.R. Bollinger, K.R. Ihlanfeldt, D.R. Bowes, Spatial Variation in Office Rents within the Atlanta Region, Urban Studies 35 (1998) 10971118. https://doi.org/10.1080/0042098984501.

[26] B. Rabia, A.C. Aydinoglu, Providing land value information from geographic data infrastructure by using fuzzy logic analysis approach, Land Use Policy 78 (2017) 46-60. https://doi.org/10.1016/ j.landusepol.2017.07. 029.

[27] O. Evren, V. Dokmeci, G. Kiroglu, G. Egdemir, Spatial Analysis of Residential Prices in Istanbul, European Planning Studies, 15 (5) (2007) 707-721. https://doi.org/ 10.1080/09654 310701214085.

[28] N.D. Tho, N.T.M. Trang, Researching Marketing Sciences - Applying SEM linear structure model in business administration, Publisher of National University of Ho Chi Minh City, 2007 (in Vietnamese).

[29] J. Nunnally, Psychometric Theory, New York : McGraw-Hill, US, 1994.

[30] H. Trong, C.N.M. Ngoc, Analyze research data with SPSS, Hong Duc Publishing House, 2008 (in Vietnamese).

[31] A.F.M. Alkarkhi, W.A.A. Alqaraghuli, Factor Analysis, Easy Statistics for Food Science with R, 143-159, Elsevier, 2019. 
[32] J.B. David, M. Knott, I. Moustaki, Latent Variable Models and Factor Analysis: A Unified Approach, Wiley, UK, 2011.

[33] S. Thomas, The analytic hierarchy process, MCGraw-Hill, New York, 1980.

[34] S. Thomas, The analytic hierarchy process- What it is and how it is used, Mathl Modelling 9 (1987) 161-176. https://doi.org/10.1016/0270-0255(87) 90473-8.

[35] T.Q. Binh, L.P. Thuy, Đ.T.M. Tam, Researching to build grassroots land information system software in urban areas (case study of Nguyen Du ward, Hai Ba Trung district, Hanoi city), Project VNU - QG.08.14, 2010 (in Vietnamese).

[36] L. Binbin, M. Charlton, A.S. Fotheringhama, Geographically Weighted Regression Using a Non-Euclidean Distance Metric with a Study on London House Price Data, Procedia Environmental Sciences 7(2011)92-97. https://doi.org/10.1016/ j.proenv. 2011.07.017.

[37] A.S. Fotheringham, C. Brunsdon, M. Charlton, Geographically Weighted Regression-The Analysis of Spatially Varying Relationships, Wiley, University of Newcastle, UK, 2002. 